\section{Die große Tiefenwirkung und lange Wirksamkeit des Jodanstrichs.}

Von J. Schunacher in Berlin.

Woranf die Wirkung der Jodtinktur bernlit, ist e'ine noell nicht ganz entschirdens Frage. Fcist nud Bonhoff ${ }^{1}$ ) nuterscheiden bei der anBerlichen Alıwendung $\overrightarrow{a d e s}$ Jods eine angenblickliclie, prinuär' und rinc rinige Zeit anhaltende, sekundäre Wirkung, dic durch dic entstehenden organisclien Jodverbindungen bedingt werde. Anf Gr'und meine'r diesbezïglichen, weiter unten zu schildernden Untersucliungen 1111 ich dieser Ansicht beipflichten.

Ich gestehe ein, daß ich mir fruher niclit gnt erklär',n kounte, warnull eine beginnonde Zahnperiostitis oder ein beginnender Furnulkel durch einc Jodpinselıng am Ausbrnch verhindert wurde. Manl exklart dies lıăfig damit, daß durch deı chemischen Reiz eine Hypurämic cutstunde, die alsdann ihren heilsamen Einfluß anf den Krankhcitsproze $\nmid 33$ ausibre. Alıdere sagtell, es ist eben die Tief $n$ wirknng des Jods. Wie soll abe') ein so reaktionsfähiger Körper wic das Jod an die Ntellen de' Infektion gelangell, die von ihm durch brcite Eiwcißschichten getrenut sind? Dicse Frage zu entscheiden, interessierto micl vor allenl.

Dub es das freie Jod nicht sein kann, wie von verschiedenen Seitell ange nommen wird, darmber kann inan nur einer Meinung sein. Sekundar kanı einc Abspaltning freien Jods aus Jodeiweißverbindunge'n vielleicht (rfolgenl. Das Nachistliegende ist, inzunehilell, daß sich das Jod unte'l Halogenierung der Eiweißkorper' bindet und daß diese alsdanll cesinfizicrende Lig'nschaften entfalten. Daß Todeiweißverbindungen desinfizicercude: nnd entwicklıngshonnul'nde Eigense'haften zukommen, ist lekanit und kounte ieh r.benfalls nachweisen.

Man kann sich davon ohne weiteres uberzengern. Man stellt sich 'nuc' wäBrige Ennulsion einer' Kultur' volı Bacillns pyogenes anleus he' Mit zwe Tropfen dieser Emulsion inpft man 10 con eines eben hei ('twa 40" noch flussigen Agarnährbodens. Nach 24 Ninnden sind anf ler gegossenc'n Platte; unzählige Kolonien anfgegangen.

Anders auf einen jodierten Nahrboden. Zn 5 Tropfen offizineller lodtinktur braehte: icl novifl von dem obigcil Nährboden, bis alles . lod gebunden war und Stärkekleister kein freics Jod melir anzeigte. Dar.u waren 15 enn Nahrboden elforderlich. Wurde dann dirser Nabrhode 11 wie oben hehandelt. so war ein Wachstiln nicht zil konstatier'en.

Ire hei der Jodiernug entstandenen organischen Jodeiweißverbilldungen mússern alsdann dureh den Säftestrom alluáhlich, ohn eine - he nische Unuandlung dabei zu erfahren, zil den tieferen Zellwhichten gelangen. wie wir dies von 'der Tiefenwirkung des Hollen" steins her wissen. Bei den Nohleinhauten wird dieser Vorgang ans antatonische'11 Grinden rascher verlanfen nuld se'in Einflul bo'deutender sein. Nin ist es garnicht einfacl, den exaktell Beweis fill das Vorhandensen ion Jodeiweißverbindungen in der intakten Haut zu führen. [ch silehte die Frage auf folyende Art \%n entscheiden. Behandelt nian "Jodeiweils, hergentellt dureh .Todierung ejner wäBrigen Hülnereiweißlosung nit riner waßrige'n Lösing von ,Jodsánre. so scheidet sich freies Jod ab. Jann mußte nlan anch anf diese Weise dif .Jodenweißrerbindungen 11 der intakten Haut llachweisen konnen.

Ich jodierte zu diesen Zweck eine Hautstelle und ließ das .Jod f.mige Stunder einwirken. Alsdanı behandelte ich die betreffeside itelle einige Minuteı lang nut einer $10 \%$ igen Natriumthiosulfatlösung, his jede erkennbare .Jodfarbe verschwunden war, wobei bekanntlich das .Jod nuter. Bildıng tetrathionsuuren Salzes gebunden wird. Die lctzten Reste freien Jods sucht: ich dadurch zu beseitigen, daß ich vor' tustellung des Versichs funf Stundeti verstreichtin ließ. Behandelte ich diese Stellen alsdann mit der Jodsänlelosung, so entstand pronpt an der so beliandelten Stelle, wieder cill Jodanstricl, als Zeichenl, daB hicr Jodeiweißverbindungen zungegen waren. Naturgemäß ist der Jodanstrich nicht so intensiv wis fl'mher.

Spluren freien Jods, die theoretisch noch in der Haut vorhanden Neilı kónnten, vermogen nit Jodsäure nicht zll reagieren. Denı Eillwand, daB noch intaktes Jodkalium²) zingegen gewesen sein kann, das alsdann mit der Jodsäıre wieder reagierte, ist entgegenzuhalten, da $\beta$ das Experinent auch mit gewohnlicher Jodtinktur gelingt. Dem Einwand, daß sich die freie Jodsäure inter Abscheidung freien Jods sehr leicht mit organischen Kórpern zersetzt, ist entgegenzulialten, daß man durch einen einfachen Jodsamreanstrich all einer nicht vorbehandelten Stelle kein freies Jod er'zeugen kann.

1) M. m. W. $1915 \mathrm{Ni} .4$

2) bei Jodierung mit naszierendem .Jod.
Uebcrrasehent int die lange 'Jeit, nach der es nocl gelingt, dis Jodeiweißverbindunge n nachznweisen. Jodient nall die Haut in Form irgendeiner geonetrischen Figur, beispielsweise in Form eines Quadrats, so kanll niall nacli dei T'age'n nocli git das erzengte geometrisehe Gcbildc wicder auffinden, wenll moll die hctreffende (ígend mit piner Jodsäurelosung uberpinselt, \%11 cill Zeit, wo längst nichts mehr von dew ursprïnglichen Jodanstrich $\%$ sehen ist. Urborall da, wo seiner\%eit die Jodierning crfolgte, sind also noch Jodeiweißvcrbindungen *ugegen. is selang all dem Jodanstrich etwas gcinacht wiurde, noch aclit Tage sjäter c'inen schwach gelben, aber deutlichen Jodstreifen mit Jodsäurelösung \%11 crocugen. Mall muß bei diesem Experiment natürlich Stellen wählen, dic nicht so lä̈nfig wic die Hände mit Seife und Handtuch in Berührinls kommen, beispiclsweise dcı Unterarm.

Da wir wissell, daß täglich eine nicht unbcträchtliche solicht der Epidermis verloren geht, und wir nach acht Tagen noch in der volr inucn sich regenericrenden Epidermis Jodeiweißverbindungen nachweisen konnen, so inuß der ursprüngliche Jodanstrich seinen Einflul, bis in sehr ticfe Hautstellen geltend gemacht haben.

In hohem Grade walirscheinlich ist es, da Jodeiweißverbindungen leicht mit Säuren freies Jod liefcrn, daB dies auch die ill der Haut vor. kommenden organischen Säuren zun Teil vermögen. 